\title{
A comprehensive evaluation of the level of noise pollution in hospitals of Kermanshah University of Medical Sciences
}

\author{
Mousavi S.A. ${ }^{1,2}$ and Sohrabi P. ${ }^{1, *}$ \\ ${ }^{1}$ Department of Environmental Health Engineering, Kermanshah University of Medical Sciences, Kermanshah, Iran. \\ ${ }^{2}$ Research Center for Environmental Determinants of Health (RCEDH), Kermanshah University of Medical Sciences, Kermanshah, Iran. \\ Received: 31/12/2016, Accepted: 17/02/2018, Available online: 23/07/2018 \\ *to whom all correspondence should be addressed: e-mail:psohrabi23@yahoo.com
}

\begin{abstract}
Noise pollution higher than the standard values intensifies the patients' disease and also has negative effects on hospital staff. This study aimed to determine the level of noise pollution in the Kermanshah hospitals and also to compare obtained data with national and international standards. Multifunctional sound level meter (Model CEL450), has been applied for determining the sound level in different parts of a hospital in 3 different time of day (morning, visiting hours (evening), and night) for working day and holiday. The highest level was on working days and it was $60.24 \pm 5.76 \mathrm{~dB}$. The average noise level on holiday was $58.15 \pm 5.44 \mathrm{~dB}$. Generally, the average noise level in all studied hospitals was higher than the standard levels. The results of the analyses showed that when the $P=0.003$, there's a significant difference between the average noise levels on different days. According to the results of the studies, it is necessary to plan and take managerial and technical - engineering measures to reduce the noise levels to standard levels.
\end{abstract}

Keywords: Noise, Noise pollution, hospital, Kermanshah,

\section{Introduction}

Today in many countries the issue of noise pollution among the different types of environmental pollutions has been identified as a widespread and even global problem (Barrientos et al., 2004). The World Health Organization has introduced the noise pollution in large cities as the third most dangerous pollution which is mentioned after the air and the water pollution (Mohammadi, 2009). The noise pollution is a level of sound that causes harassment, damage and provoke human. This kind of sound is annoying and unwanted and doesn't have steady weight. It should be noted that these sounds don't have pleasant harmony and cause irritation. Moreover, it can also have significant detrimental effects (Joneidi Jafari et al., 2014). Since facing with excessive noise reduces health and in general has negative effects on all living organisms, therefore it is considered as one of the environmental pollutions (Heydari et al., 2012). Hospital is one of the most important institutions for providing health services that plays an important role in restoring physical and spiritual health to the patients, in medical researches and also in training the required forces of health and medical groups using its facilities, so one of the major issues in this place is having quiet environment. In addition, the staffs need a quiet environment for ideal serving and they consider that the comfort of patients during the hospitalization is very important in their recovery process (Jafari et al., 2013).

It has been reported that the effects of noise in the hospital environments are perturbation in conversations of staffs and patients, sleep perturbation in patients and reducing the recovery time for patients (Karami et al., 2012). It was found that the sudden increase in noise can cause physiological reactions such as increasing blood pressure, increasing heart rate and increasing breathing frequency. Also, the evidence indicates that continuously working in an environment with high noise causes many neurological and psychiatric disorders and other problems such as excessive fatigue, depression, irritability and mental fatigue. Also, sound had indirect effects on human performance such as reducing efficiency and productivity, increasing the risk of accidents and errors because of lack of concentration and reducing the personnel accuracy. Sound acts as a risk factor that causes medical and nursing errors (Golmohamadi et al., 2010; Cmiel et al., 2004; Penney et al., 2004). Ulrich et al., (2004) studied 130 scientific papers and reported that the noise pollution is a major stressful factor for patients and hospital staffs. The findings of this study showed that in the health centers, reducing the noise pollution increases the patient satisfaction from provided services, improves the quality of their sleep and decreases their blood pressure; also, the employees have higher effectiveness and efficiency (Urich et al., 2004).

By determining the sensitiveness and importance of the noise pollution in the past decades, many studies were allocated to assess the sound pollution in different parts of the hospital and numerous reports indicate that the noise were higher than the studied limit. Poursadegh et al., (2001) investigated the level of sound in Imam Reza and Qaem hospitals in Mashhad city, Iran. Their results showed that the noise pollution is higher than the authorized limit in most parts of the hospitals (Poursadegh et al., 2001). Also, in the study that was conducted by Sobotova et al., 
(2007) in hospital environments, it was found that the average equivalent noise level in the most of hospitals was higher than the authorized limit (Sobotova et al., 2007). The studies of Kracht et al., (2006) to determine the noise levels in the operating rooms of Johns Hopkins Hospital showed that the noise pressure level in orthopedic surgeries is higher than the other surgeries and it was about $66 \mathrm{~dB}$, while the noise level in other surgeries such as cardiology and urology was determined about 62 to 65 $\mathrm{dB}$ (Kracht et al., 2006). As it was mentioned the environmental noise in hospitals is considered as a stressful factor in the world and also it is considered as a danger for health and healing the patients. So, the World Health Organization (WHO) provides some instructions to improve the noise management in the community and also to reduce the effects of noise on the health of patients (Karami et al., 2012). The standard of the WHO for the level of noise in hospital rooms during day and night are $35 \mathrm{~dB}$ and $30 \mathrm{~dB}$, respectively (Cordova et al., 2013). According to the EPA standard the authorized limit of noise in the hospitals during the day is $45 \mathrm{~dB}$ and during the night is 35 dB (Kahn et al., 1998). According to Iran national standards, the limit range of noise in outdoors and indoors of the hospital during the day from 7 am to $22 \mathrm{pm}$ is respectively $55 \mathrm{~dB}$ and $45 \mathrm{~dB}$ and during the night from $22 \mathrm{pm}$ to $7 \mathrm{am}$ is respectively $45 \mathrm{~dB}$ and $35 \mathrm{~dB}$ (Kayvani et al., 2004).

Creating a relaxed atmosphere in the hospital will be beneficial both for the patient and the health care team. The people who are responsible for the health of patients in the hospital have better conditions for faster recovery of patients in the quiet environment of hospital because they are not so tired and their physical and mental stresses are less (Otenio et al., 2007). Therefore, the present study aimed to evaluate the level of noise pollution in different parts of the hospitals of Kermanshah university of medical science to use the results for planning and considering corrective actions in order to control the noise and improve the hospital environment.

\section{Materials and methods}

This descriptive - analytical study was done in Taleghani, Imam Ali, Imam Reza, Dr. Mohammad Kermanshahi and Farabi hospitals in Kermanshah city, Iran. Measuring the noise levels in different parts of hospitals was performed during two days of a week (a typical day of working week and a holiday (Friday)) and in 3 different periods including morning (8-10 am), afternoon (2-4 pm visit hours) and night $(8-10 \mathrm{pm})$. The noise in different parts of the hospital and also the environmental parameters of the noise $L_{\text {max }}$, $L_{\text {min, }}$ and $L_{e q}$ were measured by an audiometer machine model CEL - 450. In order to ensure the accuracy of the measurement results, the sound level meter was calibrated using a calibrator before starting the measurement. The height for measuring noise in different parts of the hospital on the basis of auditory height was considered 1.5 meters from the ground and in the hospital rooms was considered 1 meter. The spongy shield on the surface of the microphone was used to remove the effect of air flow and wind. The noisiest parts in each hospital were identified after measuring and collecting data and the level of noise in different part of the hospital was compared with each other and also with the WHO standards. All parts of hospitals were studied and evaluated in terms of noise pollution. After collecting data, the results were analyzed through ANOVA using SPSS software version 21. The meaningful relationships between variables and acceptance or rejection the proposed hypotheses were tested.

\section{Results and Discussion}

According to figure 1 , the results showed that the emergency department in Taleghani hospital has $68.5 \mathrm{~dB}$ of average noise level in the evening of weekdays and holidays. In the mornings of the weekdays the clinic and emergency department were the most crowded sections with respectively $71.7 \mathrm{~dB}$ and $70.1 \mathrm{~dB}$ of average noise level. The public meeting ward and the hallway on meeting hours during the weekdays and the woman's ward and the hallways on holidays were the most crowded wards in the hospital. The average noise in the morning was more than the average noise in the afternoon which is because of too many visitors, clienteles and patient's family in different wards of the hospital, so their communications make too much noise (Table 1). In Imam Ali hospital and during the meeting hours, the most crowded ward is the hallway in weekdays and the wards are the most crowded parts of the hospitals on holidays. In the evening hours of holidays, the emergency department is the most crowded part and also in the evening hours of weekdays, the hallway and the emergency department are respectively the most crowded parts of the hospital. The most crowded parts of the hospital are clinic in weekday mornings and the emergency department and the hallway radiology in holiday mornings (Table 1). The hallway and the emergency department are the most crowded parts of the Imam Reza hospital during night shifts of holidays and weekdays. In visiting hours, the entrance hallway, the wards and the woman hospitalization wards were the most crowded parts of the hospital. In the holiday and weekday mornings, the hallway and the emergency department were respectively the most crowded parts of the hospital. In Dr. Mohammad Kermanshahi hospital during the weekday nights and holidays, the hallways, ICU and the emergency department were the most crowded parts and in visiting hours of weekdays, the hallways, the emergency department and the neonatal hospitalization were the most crowded parts and in visiting hours of holidays, the hallways, the neonatal hospitalization and the general admission were more crowded. In morning hours of weekdays, the clinic, the hallway and the neonatal hospitalization were the most crowded parts of the hospital while in holiday mornings, the hallway, the laboratory and the radiology were the most crowded parts. In Farabi hospital, the ICU, the emergency department and the hallway during night shifts of the weekdays and the ICU and the hallways during night shifts of the holidays were the most crowded parts of the hospital. In visiting hours of weekdays and holidays, the ward was the most crowded part of the hospital. In weekday mornings the most crowded parts were administrative departments and the clinics. The ICU and 
the emergency department were the most crowded parts during holiday mornings. As it can be seen in Figure 2, the total noise average in Taleghani hospital is $10.32 \pm 63.71$, in
Imam Ali hospital is $9.43 \pm 600.34$, in Imam Reza hospital is $9.61 \pm 59.19$, in Dr. Mohammad Kermanishahi hospital is $9.91 \pm 57.48$ and in Farabi hospital is $8.85 \pm 57.7$.

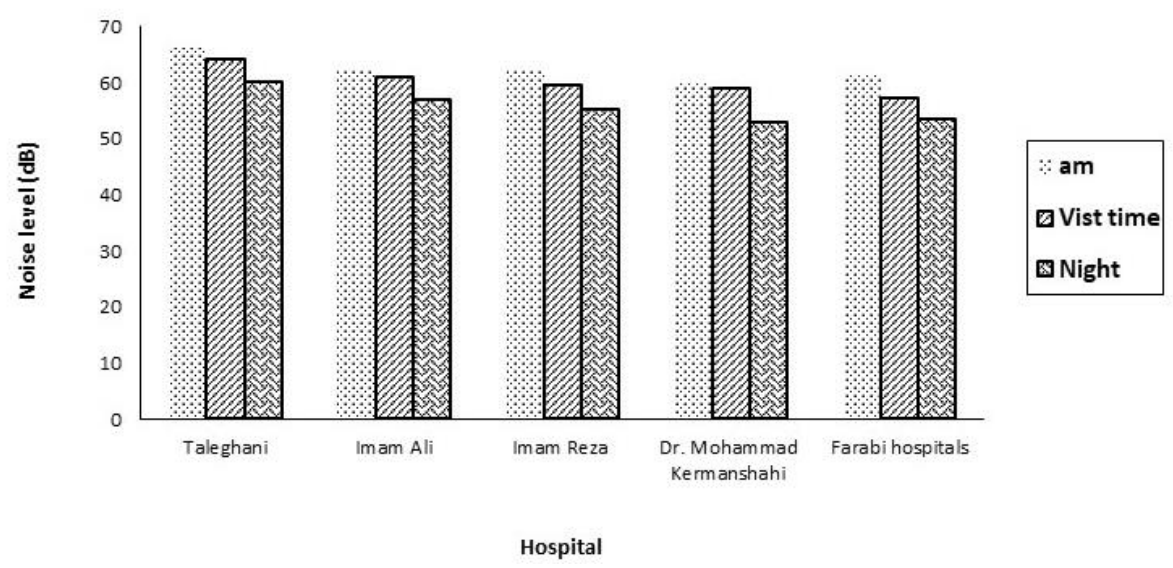

Figure 1. The overall average noise level $(\mathrm{dB})$ at different hours of working time in hospitals

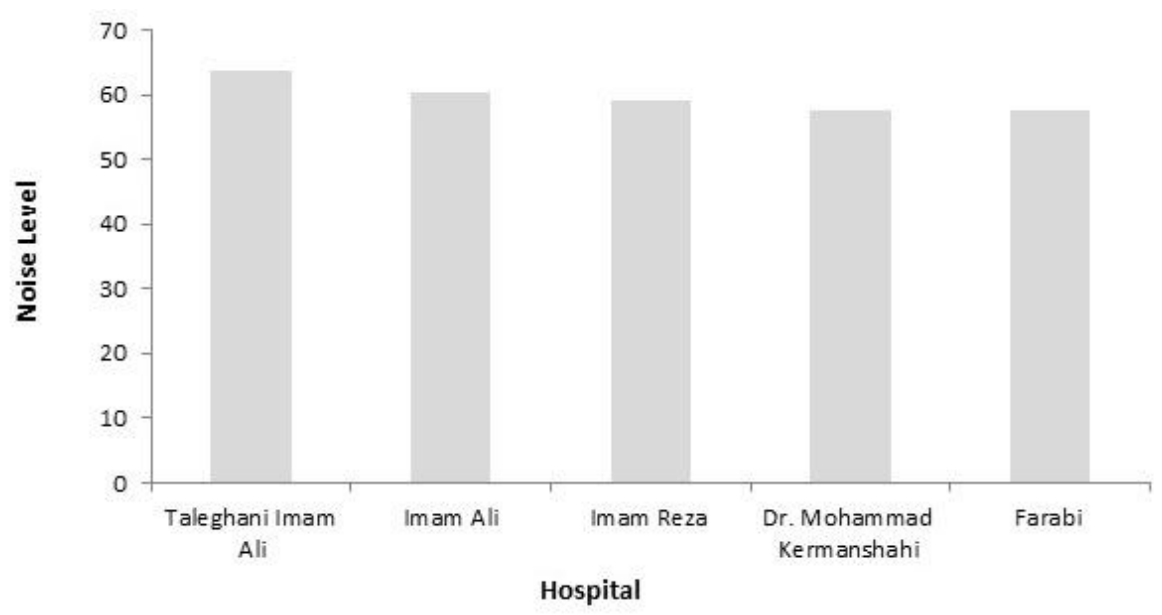

Figure 2. The overall average noise level $(\mathrm{dB})$ in hospitals

Table 1. Compares the average noise level in working days and holidays in hospitals

\begin{tabular}{lcccc}
\hline \multicolumn{1}{c}{ Weekdays } & \multicolumn{2}{c}{ Working day } & \multicolumn{2}{c}{ Holidays } \\
\hline $\begin{array}{l}\text { Noise level } \\
\text { Hospital }\end{array}$ & Mean & S.D & Mean & S.D \\
\hline Taleghani & 64.48 & 9.45 & 62.85 & 11.23 \\
\hline Imam Ali & 61.41 & 9.81 & 59.08 & 8.87 \\
\hline Imam Reza & 60.5 & 9.6 & 57.67 & 9.47 \\
\hline Dr. Mohammad Kermanshahi & 58.36 & 10.3 & 56.52 & 9.44 \\
\hline Farabi hospitals & 59.09 & 9.39 & 56.08 & 7.94 \\
\hline
\end{tabular}

Studying the authorized limit of noise pressure level in hospitals during day $(45 \mathrm{~dB})$ and at nights $(35 \mathrm{~dB})$ showed that this value is higher than the authorized limit (Bharathan et al., 2007).

Studying the different parts of the hospitals showed that the emergency department and clinic were the most crowded parts of the hospitals in the morning which is because of overcrowding in all parts of the hospitals. These results completely are corresponded with the studies of Heidari et al., (2012) in Qom and the studies of Karami et al. (2012) in Ahvaz. Also, the studies of Bharathan et al.,
(2007) showed that the noise levels in different parts of an educational hospital were higher than the threshold patients and visitors and also because of haunting their companions in these parts of the hospital. The studies of Movafagh et al., (2009) on noise pollution in hospitals of Tehran showed that the noise pressure level in various parts (including the waiting room, clinic and the emergency department) was higher than the authorized limit and it is consistent with the results of this study (Moaffagh et al., 2009). Also, during the meeting hours, the clinical departments were the most crowded parts of the hospitals. 
The results of estimation of noise levels during different days showed that in all hospitals the highest noise levels were on weekdays. The average noise on weekdays was $5.76 \pm 60.24$ and on holidays was $5.44 \pm 58.15$. Generally, there is a significant difference in average noise levels during different days ( $P$ Val $=0.003$ ). According to the analysis of variance in total noise levels during different time periods, there is a significant difference in different parts of the hospitals ( $P$ Val $=0.000)$.

These results are consistent with other national and international studies that have been published in this field (7, 18-19). For instance, a study was done by Amoei et al., in Babol (2014). According to the partial correlation coefficient, there is a significant relationship between working days $(\mathrm{P} \vee \mathrm{Val}=0.001)$ and the volume and also between the volume and different working shifts ( $P$ Val $=0.000)$. The results showed that Taleghani hospital has the highest average noise level among the other hospitals. This result wasn't unexpected because of the type and the nature of provided services in this hospital.

In general, and according to the results of studied hospitals, the noise level was higher than the provided standards-by the World Health Organization-during weekdays and holidays and in each period including morning, the meeting hours and evening. So, these results are consistent with the results of other researchers, including the studies of Karami et al., (2014) in Ahvaz.

It is necessary to plan and to take managerial and technical-engineering measures to reduce the noise level to the standard authorized limits because the noise pressure level in the hospitals of Kermanshah is high, also because the noise has damaging effects on desirable results in improving patients and the personnel should feel comfortable in order to serve desirable. Therefore, increasing the awareness of employees and managers of hospitals and also the society about the importance of holding down the noise pollution level, setting managerial rules and regulations about the way that the personnel and the other people move in the hospital environment, creating warning signs to keep the wards silent and also verbal warnings to patients, companions of patients and staffs can help a lot to control the noise pollution in hospitals.

\section{Conclusion and technical comments}

In order to reduce the noise pollution, rehabilitation of hospital environment or displacement and modernization of them is recommended. Also using engineering and technical principles can considerably reduce the noises inside the hospital. For example the air conditioning system can be repaired, the sound-absorbing materials (Acoustic: absorbents with high reducing coefficient) can be used in the ceiling, floor and the wall of the hospital, using materials such as stone in the walls and floors that causes echo in the wards or in the hallways should be avoided, special shoes which don't produce sounds should be used for employees, the transportations should be maintained and lubricated (trolley), the audio systems (pager) should be changed and the sound insulation systems should be used too.

\section{Acknowledgments}

This work was supported by Kermanshah University of Medical Sciences. The author would like to thank faculty of health for preparing equipment.

\section{References}

Aaron J.N., Carlisle C.C. and Carskadon M.A. (1996), Environmental noise as a cause of sleep disruption in an intensive care unit, Sleep, 19, 707-710.

Asgharnia H.A., Tirgar A, Amouei A., Fallah S.H., Khafri S., Mohammadi A.A., Peykarporsan F., Rahimi D., Beykaei S.B. and Shirkhani Z. (2014), Noise pollution in the teaching hospitals of Babol (Iran) in 2012, J Babol Univ Med Sci, 16(4), 64-69. [in Persian]

Barrientos M.C., Campbell-Lendrum D.H. and Steenland K. (2004), World Health Organization, Protection of the Human Environment. Occupational noise: assessing the environmental burden of disease from work-related impairment at national and local levels. Geneva: World Health Organization.

Bharathan T., Glodan D., Ramesh A., Vardhini B., Baccash E., Kiselev P. and Goldenberg G. (2007), What do patterns of noise in a teaching hospital and nursing home suggest?, Noise Health, 9(35), 31-34.

Cmiel C.A., Karr D.M., Gasser D.M., Oliphant L.M. and Neveau A.J. (2004), Noise control: a nursing team's approach to sleep promotion, American Journal of Nursing, 104(2), 40-48.

Cordova A.C., Logishetty K., Fauerbach J., Price L.A., Gibson B.R. and Milner S.M. (2013), Noise levels in a burn intensive care unit, Burns, 39(1), 44-48.

Golmohamadi R. and Aliabadi M. (2010), Noise Pollution and its Irritating Effects in Hospitals of Hamadan, Iran, Journal of Health System Research, 7(6), 958-964. [in Persian]

Heydari H.R., Mohebi S., Paidari N., Ramouz P., Nayebi T., Omrani D. and Rahimifard H. (2013), Noise exposure assessment among nurses in Qom Educational Hospitals in 2012, Iran, Qom Univ Med Sci J, 7(6), 46-53. [in Persian]

Jafari N., Bina B., Mortezaie S., Ebrahimi A. and Abdolahnejad A. (2013), Assessment of Environmental Noise Pollution in Feiz Hospital Wards and its Adjacent Area, Journal of Health System Research, 8(3), 377-384. [in Persian]

Joneidi Jafari A., Sahebdel E., Hajipour A., Jafari Javid M., Mireskandari S., Karvandian K., Jafarzadeh A. and Samadi S. (2014), Mean sound level in operation rooms in a referral hospital: a brief report, Tehran Univ Med J, 71(11), 739-744. [in Persian]

Kahn D.M., Cook T.E., Carlisle C.C., Nelson D.L., Kramer N.R. and Millman R.P. (1998), Identification and modification of environmental noise in an ICU setting, Chest, 114(2), 535-540.

Karami K.H., Torabipour A., Jamali N. and Jamshidi Z. (2012), Noise pollution levels in different Wards of Golestan and Fatemeh Zahra Hospitals, Iran, Scientific Medical Journal of Ahvaz Jundishapur University of Medical Sciences, 4(4), 77-84. [in Persian].

Kayvani N. (2004), National Environmental Protection Organization. Environmental Criteria and Standards. 1st ed. Tehran: Dayereh Sabz Publication; p. 13-4.

Kracht J., Busch-Vishniac I.J. and West J.E. (2006), Operating Room Noise at Johns Hopkins Hospital, J Acoust Soc A, 119(5), 3385. 
Moaffagh A., Jalilzadeh R., Delbari A. and Doosti M.R. (2009), Noise pollution levels in one of the hospitals in Tehran (Case study, Alghadir hospital). Third Expert Conference on Environmental (IRAN), Available from: http://www.civilica.com/Paper-CEE03-CEEO3 171.html. [in Persian]

Mohammadi G.H. (2009), An Investigation of Community Response to Urban Traffic Noise, Iran J Environ Health Sci Eng, 6(2), 673-680. [In Persian].

Otenio M.H., Cremer E. and Turini Claro E.M. (2007), Noise level in a 222 bed hospital in the 18th health region-PR, Braz J Otorhinolaryngol, 73(2), 245-250.

Penney PJ, Earl CE, Penney PJ, Earl CE. (2004). Occupational noise and effects on blood pressure: exploring the relationship of hypertension and noise exposure in workers, AAOHN Journal, 52(11), 476-80.

Poursadegh M., Rezaei M. and Hashemi E. (2001), A Study of Noise Pollution in Emam Reza and Ghaem Hospitals, Med J Mashad Univ Med Sci, 44(1), 8-18. [in Persian]

Sobotova L., Jurkovicova J., Stefanikova Z., Sevcikova L. and Aghova L. (2007), Noise Levels in the Hospital Environment and Health Risks, Epidemiology, 18(5), 69-73.

Urich R.S., Quan X., Zimring C.P., Joseph A. and Choudhary R. (2004), The Role of the Physical Environment in the Hospital of the 21st Century: A once-in-a-lifetime opportunity. Concord, CA, Report sponsored by The Robert Wood Johnson Foundation and The Center for Health Design. Available from http://www.healthdesign.org/chd/research/role-physicalenvironment-hospital-21st-century?page=show. Date access: 13/02/2011. 